Article

\title{
Quasi-Static Load Sharing Characteristics of a Planetary Gear Set with Planet Journal Bearings
}

\author{
Zhiqiang Guo ${ }^{1}$, Shenlong $\mathrm{Li}^{2}$, Wei $\mathrm{Wu}^{1, *}$ and Liuyang Zhang ${ }^{1}$ \\ 1 National Key Laboratory of Vehicular Transmission, Beijing Institute of Technology, Beijing 100081, China; \\ gzqiang1008@163.com (Z.G.); zly1126@hotmail.com (L.Z.) \\ 2 China North Vehicle Research Institute, Beijing 100072, China; lishenlong2000@outlook.com \\ * Correspondence: wuweijing@bit.edu.cn; Tel.: +86-10-68914786
}

Received: 29 November 2019; Accepted: 18 January 2020; Published: 7 February 2020

\begin{abstract}
The quasi-static load sharing characteristics of a planetary gear set with planet journal bearings are numerically investigated. The application of journal bearings instead of rolling bearings in planetary gear sets is an alternative to increase the rotation speed. Therefore, an effective analysis method is needed to estimate the dynamic performance of a planetary gear set with planet journal bearings. Here, an available load sharing factor computation method is developed considering the effect of the variable journal bearing stiffness. Results of an experiment are used to validate the effectiveness of the computation method. Furthermore, the load sharing characteristics of a planetary gear set with planet journal bearings are influenced by bearing parameters and operating conditions significantly due to the changing bearing stiffness of journal bearings. The effects on the load sharing factor and the bearing stiffness are studied in detail simultaneously based on the proposed analysis method. The load sharing factor of a planetary gear set with planet journal bearings has a similar variation with the bearing support stiffness under the same load. These researches provide a theoretical basis for the application of journal bearings in planetary gear sets.
\end{abstract}

Keywords: planetary gear; journal bearing; load sharing; stiffness; high speed

\section{Introduction}

In planetary gear sets, multiple planet gears are used to form power split and planetary gears provide higher power density than parallel axis gears [1]. The planetary gear set has been widely applied in wind turbines [2] and automatic transmissions [3,4]. It uses three or more spaced planet gears. In the ideal situation, each planet gear equally shares the load. However, planet gear loads are not always equally distributed in practice. With unequal loads, planet-bearing forces increase, leading to reduced bearing life and failures [5,6]. Further, the planet-bearing often operates at a high rotation speed to satisfy a higher power density requirement $[7,8]$. This also affects the planet-bearing reliability. Due to better performance at a high rotation speed [9], the journal bearing becomes an appropriate planet-bearing choice, as shown in Figure 1.

Load sharing in planetary gear sets has been investigated extensively. Hidaka [10] indicated that floating central members could lead to perfect load sharing in a three-planet gear set. In 1986, Hayashi [11] showed that load sharing characteristics are improved with the increasing input torque by an experimental study. The shear strain of planet pins was measured to investigate the load sharing characteristics. The studies mainly focused on the three-planet system. In 1994, Kahraman [12] established a nonlinear time-varying dynamic model and quantitatively investigated the influences of the main design parameters, manufacturing, and assembly errors on load sharing conditions. The load sharing characteristics of the four-planet system were investigated. A reasonable agreement between the mathematical model and the test results was achieved [13]. Kahraman also established 
a dynamic planetary transmission model [14], by which the design parameters could be given to an arbitrary number of planets. The four-planet system research was also expanded by adding a flexible ring gear [15]. Bodas and Kahraman [16] investigated the influence of manufacturing errors using an improved contact model. Singh [17] and Fyler [18] indicated that the stiffness of the needle bearings has a major influence on gear contact pattern, and the double pinion planetary arrangement is more likely to result in off-centered loading. Singh [19] also physically explained the basic mechanism of unequal load sharing and obtained the analytical expressions to estimate unequal load sharing caused by position errors. On this basis, Singh [20] proposed the concept of a load sharing map to describe the load sharing characteristics of planetary gears with arbitrary position errors and load levels. Alfred [21] designed a new low spring constant planet pin that was shown to have significantly superior load sharing characteristics. Considering the instantaneous geometric shapes influenced by tooth deformations and errors, $\mathrm{Gu}$ and Velex [22] illustrated the influence of planet position errors on the load sharing characteristics.

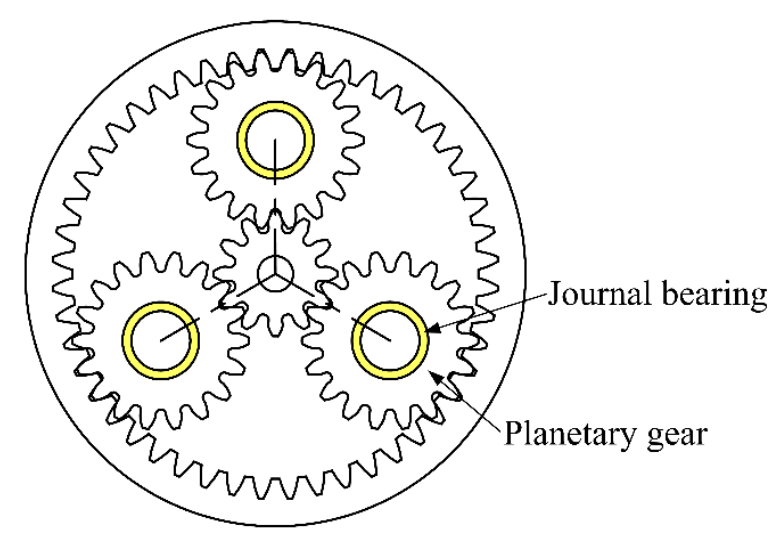

Figure 1. Configuration of a planetary gear set with journal bearings.

Most of the investigations focused on the load sharing characteristics of planetary gear sets with planet rolling bearings. The bearing stiffness can be considered as a constant. However, for the planetary gear set using journal bearings, the bearing stiffness becomes variable. It leads to more complicated load sharing characteristics. The paper studies the load sharing characteristics of planetary gear sets using planet journal bearings. A load sharing analysis model considering the hydrodynamic effect of the journal bearing is established. The influences of bearing parameters on load sharing characteristics are analyzed. It is aimed to provide an effective method for the design of the high-speed planetary gear set using planet journal bearings.

\section{Materials and Methods}

For hydrodynamic journal bearings using an incompressible lubricant of constant viscosity and operating under steady-state conditions, the general two-dimensional form of the Reynolds equation is given by [23]

$$
\frac{\partial}{\partial x}\left(h^{3} \frac{\partial p}{\partial x}\right)+\frac{\partial}{\partial z}\left(h^{3} \frac{\partial p}{\partial z}\right)=6 U \eta \frac{\partial h}{\partial x}
$$

where, $p$ is the pressure, $h$ is the oil film thickness, $\eta$ is the lubricant viscosity, $U$ is the tangential speed, $x$ and $z$ are the coordinates of journal circumferential direction and axial direction, respectively.

Figure 2 presents the planet journal bearing inside a planet gear set. The radii of the planet gear shaft and planet gear are $r$ and $R$, respectively. The center of the planet shaft $O_{1}$ is considered as the center of the bearing journal. Due to the loaded force, the center of the planet gear moves to $\mathrm{O}_{2}$, which is also considered as the center of the bearing bushing. The distance between the two centers is defined as the eccentricity, denoted by $e$. In addition, $\theta$ is defined as the eccentric angle and $\omega$ is defined as the relative rotation speed of the planet gear and the planet gear shaft. 


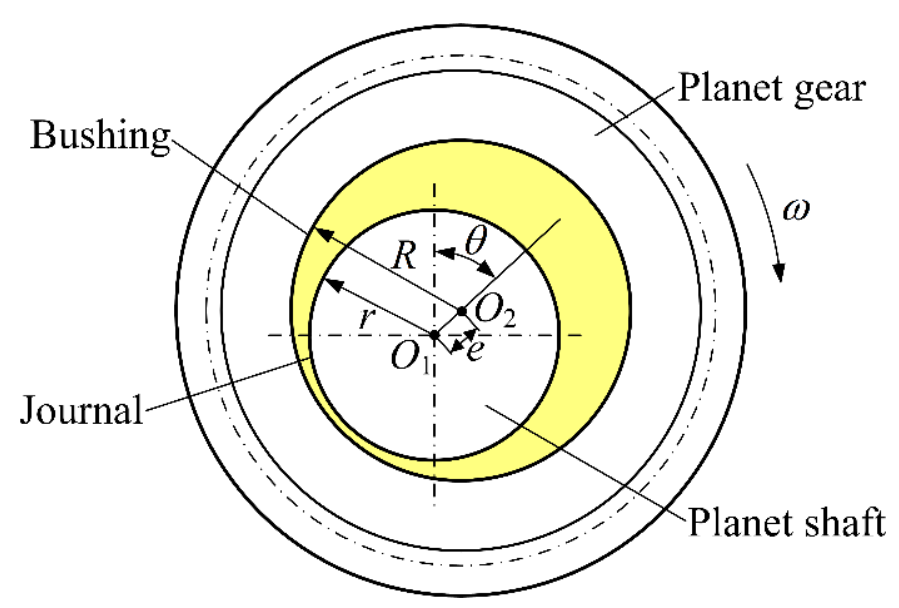

Figure 2. Parameters of the planet journal bearing.

For further analysis of the journal bearing characteristics, the coordinate transformation method is used to make all the parameters in Equation (1) be dimensionless. Equations (2)-(8) give the expressions of the dimensionless parameters:

$$
\begin{gathered}
\varphi=x / r, \\
\lambda=y /(L / 2), \\
H=h / c=1+\varepsilon \cos \varphi, \\
c=R-r, \\
\varepsilon=e / c, \\
P=p / p_{0}, \\
p_{0}=6 U \eta r / c^{2},
\end{gathered}
$$

where, $\varphi$ is the azimuth angle in the circle, $L$ is the journal bearing length and $\lambda$ ranges from -1 to $1, c$ is the original radial clearance between the bushing and the journal, $\varepsilon$ is the eccentricity ratio, $p_{0}$ is the designed pressure.

Then, the nondimensional Reynolds equation can be expressed as

$$
\frac{\partial}{\partial \varphi}\left(H^{3} \frac{\partial P}{\partial \varphi}\right)+\left(\frac{D}{L}\right)^{2} \frac{\partial}{\partial \lambda}\left(H^{3} \frac{\partial P}{\partial \lambda}\right)=\frac{\partial H}{\partial \varphi} .
$$

The finite difference method is used to discretize the dimensionless Reynolds equation. The oil pressure is obtained by the successive over-relaxation iteration method:

$$
A_{i, j} P_{i+1, j}+B_{i, j} P_{i-1, j}+C_{i, j} P_{i, j+1}+D_{i, j} P_{i, j-1}-E_{i, j} P_{i, j}=F_{i, j}
$$

where, $A, B, C, D, E$ and $F$ are the simplified parameters. They are calculated by Equations (11)-(16):

$$
\begin{gathered}
A_{i, j}=H_{i+0.5, j^{\prime}}^{3} \\
B_{i, j}=H_{i-0.5, j^{\prime}}^{3} \\
C_{i, j}=\frac{D}{L}\left(\frac{\Delta \varphi}{\Delta \lambda}\right)^{2} H_{i, j+0.5^{\prime}}^{3}
\end{gathered}
$$




$$
\begin{gathered}
D_{i, j}=\frac{D}{L}\left(\frac{\Delta \varphi}{\Delta \lambda}\right)^{2} H_{i, j-0.5^{\prime}}^{3} \\
E_{i, j}=A_{i, j}+B_{i, j}+C_{i, j}+D_{i, j}, \\
F_{i, j}=\Delta \varphi\left(H_{i+0.5, j}-H_{i-0.5, j}\right) .
\end{gathered}
$$

Reynolds boundary conditions are used in the calculation and adopted as

$$
P=0, \partial P / \partial \varphi=0\left(\varphi=\varphi_{2}\right),
$$

where, $\varphi_{2}$ is the angular starting position of oil film rupture along the circumferential direction [24], which can be obtained when the convergence criterion is reached.

The convergence criterion to stop the iteration is expressed as

$$
\sum_{i=1}^{m+1} \sum_{j=1}^{n+1}\left|\frac{p_{i, j}^{(k+1)}-p_{i, j}^{(k)}}{p_{i, j}^{(k+1)}}\right| \leq E R R,
$$

where, ERR is the specified error, which is $10^{-4}$ used in the calculation.

\section{Load Sharing Analysis Method}

\subsection{Planetary Gear Set Errors}

Manufacturing and assembly errors of the planetary gear set make the center distance change. It is common to allow the sun gear to float in the radical direction in the planetary gear transmissions of vehicles [13]. The sun gear was chosen as the floating component. As shown in Figure 3, in a planetary gear set, $k_{p}$ and $k_{s}$ are the support stiffness coefficients of the bearing and the sun gear. $k_{s p i}$ and $k_{p r i}$ are the meshing stiffness coefficients of the $i$ th sun-planet pair and the $i$ th ring-planet pair. $r_{b s}$ and $r_{b p i}$ are the pitch circle radii of the sun gear and the $i$ th planet.

To study the dynamics of the planet, the equivalent meshing backlash caused by the manufacturing errors [25], the assembly errors [26], and the floating component errors [13] needs to be considered. Therefore, several parts have been taken into account in the cumulative meshing error calculation, as shown in Figure 4.

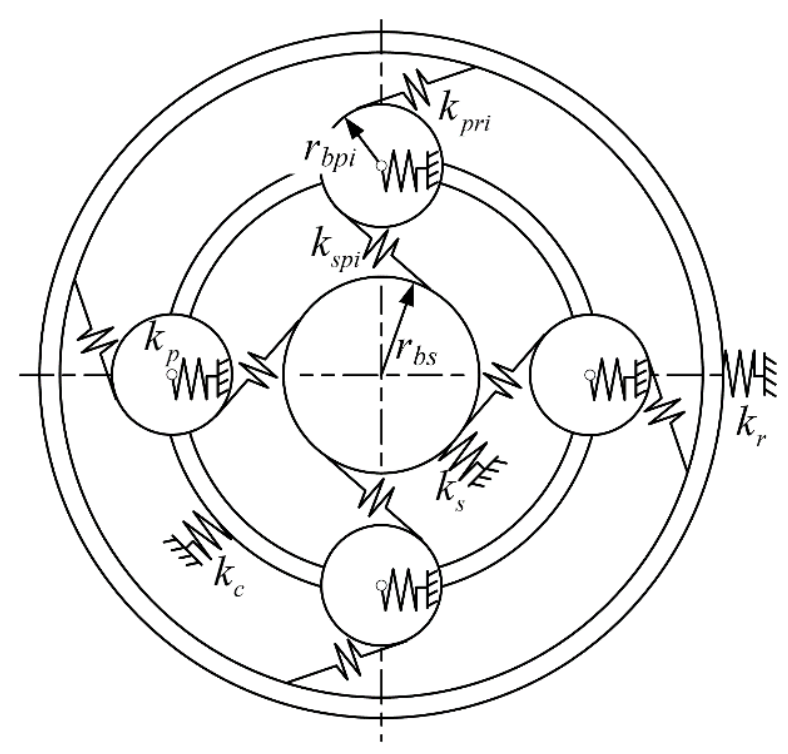

Figure 3. Model of a planetary gear set. 


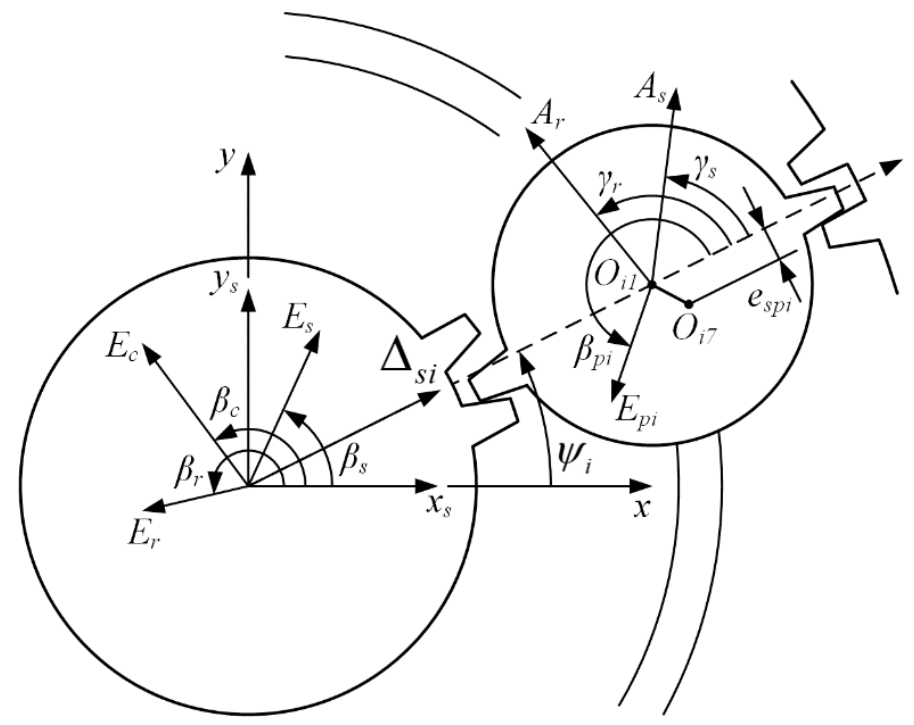

Figure 4. Schematic of floating component error and cumulative meshing error.

Because of the manufacturing error of each component of the planetary gear set and the assembly errors in the planet-sun gear pairs and the planet-ring gear pairs, the center of the $i$ th planet moves to $O_{i 7}$ from position $O_{i 1}$, and the cumulative meshing error is represented by $e_{s p i}$, as shown in Figure 4 . The manufacturing errors are composed of four parts: the sun gear, the planet, the carrier, and the ring. As illustrated in Figure 4, the manufacturing errors of the sun gear, the carrier, the ring gear, and the $i$ th planet are defined by a respective magnitude $E_{s}, E_{c}, E_{r}, E_{p i}$ [27] and a respective initial orientation angle of the high point $\beta_{s}, \beta_{c}, \beta_{r}, \beta_{p i}$. The assembly errors consist of the planet pin position error from the sun gear and the planet pin position error from the ring gear. The assembly errors of the sun-planet gear pair and the ring-planet gear pair are defined by their magnitudes $A_{s}$ and $A_{r}$ and their initial orientation angles $\gamma_{s}$ and $\gamma_{r}$, respectively.

The equivalent meshing errors caused by the manufacturing error of each component are given by

$$
\begin{gathered}
e_{E s i}=-E_{s} \sin \left[\left(\omega_{s}-\omega_{c}\right) t+\beta_{s}+\alpha_{w}-\varphi_{i}\right], \\
e_{E p i}=-E_{p i} \sin \left[\left(\omega_{p}-\omega_{c}\right) t+\beta_{p i}+\alpha_{w}\right], \\
e_{E c i}=E_{c} \sin \left(\beta_{c}-\varphi_{i}\right) \cos \alpha_{w}, \\
e_{E r i}=E_{r} \sin \left(\omega_{c} t-\beta_{r}+\alpha_{w}+\varphi_{i}\right) \cos 2 \alpha_{w},
\end{gathered}
$$

where, $e_{E s i}, e_{E p i}, e_{E c i}$, and $e_{E r i}$ are the equivalent meshing errors of the $i$ th planet. $\omega_{s}, \omega_{c}$, and $\omega_{p}$ are the rotation speeds of the sun gear, the planetary gear, and the carrier, respectively. $\alpha_{w}$ is the helix angle. $t$ is time. $\varphi_{i}$ is the nominal position angle of the $i$ th planet relative to the carrier.

The equivalent meshing errors, based on two resources of assembly errors, are expressed by

$$
\begin{gathered}
e_{A s i}=-A_{s} \sin \left(-\omega_{c} t+\gamma_{s}+\alpha_{w}-\varphi_{i}\right) \\
e_{A r i}=A_{r} \sin \left(\omega_{c} t-\gamma_{r}+\alpha_{w}+\varphi_{i}\right) \cos 2 \alpha_{w},
\end{gathered}
$$

where, $e_{A s i}$ and $e_{A r i}$ are the equivalent meshing errors of the $i$ th planet.

Therefore, the accumulative meshing error on the meshing line of the $i$ th planet-sun gear pair can be summed up by

$$
e_{s p i}=e_{E s i}+e_{E p i}+e_{E c i}+e_{E r i}+e_{A s i}+e_{A r i}
$$


The floating component error is assumed as $\Delta_{s i}$, which is a function of time. To calculate the floating error of the sun gear, variables $x_{s}$ and $y_{s}$ are introduced to characterize the displacement of the center of sun gear in two directions, shown in Figure 4. Thus, the floating component error is

$$
\Delta_{s i}=x_{s} \cos \psi_{i}+y_{s} \sin \psi_{i}
$$

where,

$$
\psi_{i}=\omega_{c} t+\frac{\pi}{2}-\alpha_{w}+\varphi_{i}
$$

\subsection{Journal Bearing Stiffness}

Equivalent meshing integrated stiffness of the planetary gear set is composed of three parts: the sun-planet meshing pair, the planet-ring meshing pair, and the planet gear supported by a journal bearing. Therefore, it is necessary to transform these three elements to the meshing line of a sun-planet gear pair. Thus, the effective planet support stiffness $k_{\Sigma}$ can be estimated as [28]

$$
\frac{1}{k_{\Sigma}}=\frac{1}{k_{p}}+\frac{1}{k_{s p}+k_{p r}} \text {. }
$$

Furthermore, the journal bearing stiffness can be significantly different under various operating conditions.

Under different loads, the center of the planet gear changes, as shown in Figure 5. As the load increases, the eccentric angle $\theta$ decreases, the planet center moves from $O^{\prime}$ to $O^{\prime \prime}$. The projection of the planet center on the load action line moves from $p$ to $p^{\prime}$. Then, the bearing support stiffness is given by [29]

$$
k_{p}=\frac{\partial W}{\partial L_{O p}},
$$

where, $W$ is the load and $L_{O p}$ is the distance between $O$ and $p$. $L_{O p}$ can be calculated by

$$
L_{O p}=e \cos \theta=c \varepsilon \cos \theta \text {. }
$$

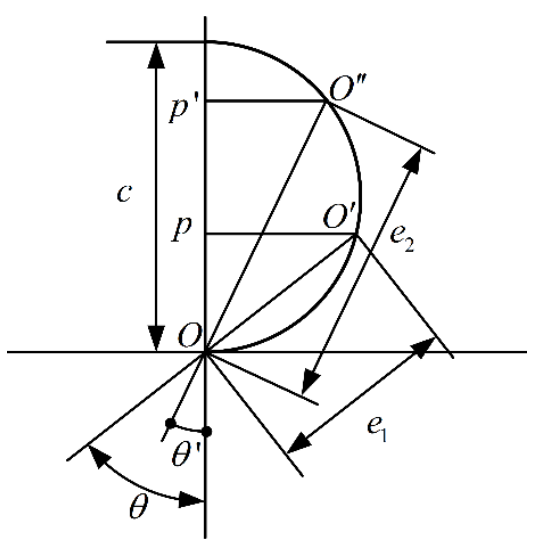

Figure 5. The variation of the planet gear center.

Substituting Equation (30) into Equation (29), the numerator and the denominator in Equation (29) are simultaneously divided by partly derivative of the eccentricity ratio. Then the bearing stiffness can be described as

$$
k_{p}=\frac{\frac{\partial W}{\partial \varepsilon}}{c\left(\cos \theta-\varepsilon \frac{d \theta}{d \varepsilon} \sin \theta\right)} .
$$


Since the dynamic pressure distribution in the journal bearing is obtained from the numerical iterative computation, the dimensional loads in both horizontal and vertical directions can be calculated by

$$
\left\{\begin{array}{c}
W_{h}=\int_{0}^{L} \int_{0}^{2 \pi r} p \cos \varphi d \varphi d \lambda=\frac{p_{0} L r}{2} \sum_{i=1}^{m} \sum_{j=1}^{n} P_{i, j} \cos \varphi_{i} \Delta \varphi \Delta \lambda \\
W_{v}=\int_{0}^{L} \int_{0}^{2 \pi r} p \sin \varphi d \varphi d \lambda=\frac{p_{0} L r}{2} \sum_{i=1}^{m} \sum_{j=1}^{n} P_{i, j} \sin \varphi_{i} \Delta \varphi \Delta \lambda
\end{array} .\right.
$$

Thus, the load is given by

$$
W=\sqrt{W_{h}^{2}+W_{v}^{2}}
$$

The eccentric angle is expressed by

$$
\theta=\arctan \frac{W_{v}}{W_{h}}
$$

\subsection{Load Sharing Factor}

When a planetary gear set with $N$ planet gears is loaded through the sun gear, the total load should be equal to $T / r_{b s}$. $T$ is the input torque. However, due to various manufacturing and assembly errors, the planet gears could not mesh simultaneously and only one planet will be loaded first. As the torque increases, the other backlash decreases, and then all the planet gears got to be loaded. During the meshing process, the rolling arc length of the sun gear along the pitch circle is given by

$$
\delta=r_{b s} \theta_{s}
$$

where, $\theta_{s}$ is the rotation angle of the sun gear.

Considering the cumulative meshing error and the floating component error, the load can be described by

$$
W_{s p i}=k_{\Sigma}\left(\delta-e_{s p i}-\Delta_{s i}\right) .
$$

Therefore, the total load can be obtained by

$$
\sum_{i=1}^{N} W_{s p i}=N \delta k_{\Sigma}-k_{\Sigma} \sum_{i=1}^{N} e_{s p i}-k_{\Sigma} \sum_{i=1}^{N} \Delta_{s i}
$$

The load above should be equal to the load on the sun gear, as demonstrated by

$$
\sum_{i=1}^{N} W_{s p i}=T / r_{b s}
$$

Substitute Equations (35) and (38) into Equation (37), as expressed by

$$
W_{s p i}=k_{\Sigma}\left[\frac{1}{N} \sum_{i=1}^{N}\left(e_{s p i}+\Delta_{s i}\right)-\left(e_{s p i}+\Delta_{s i}\right)\right]+\frac{T}{r_{b s} N}
$$

According to the stress analysis of the sun gear, the force balance equations are given by

$$
\left\{\begin{array}{l}
\sum_{i=1}^{N} W_{s p i} \cos \psi_{i}+k_{s} x_{s}=0 \\
\sum_{i=1}^{N} W_{s p i} \sin \psi_{i}+k_{s} y_{s}=0
\end{array} .\right.
$$


For an $N$-planet gear set with $N \geq 3,3 N+2$ equations can be established based on Equations (39) and (40) with $3 N+2$ variables. By calculating the load on each planet gear, the load sharing factor of the planetary gear set can be computed by

$$
\Omega=\max _{1 \leq i \leq n} \Omega_{i}=\max _{1 \leq i \leq n}\left(W_{s p i} N r_{b s} / T\right) .
$$

The flowchart of the load sharing factor calculation procedure outlined above is illustrated in Figure 6.

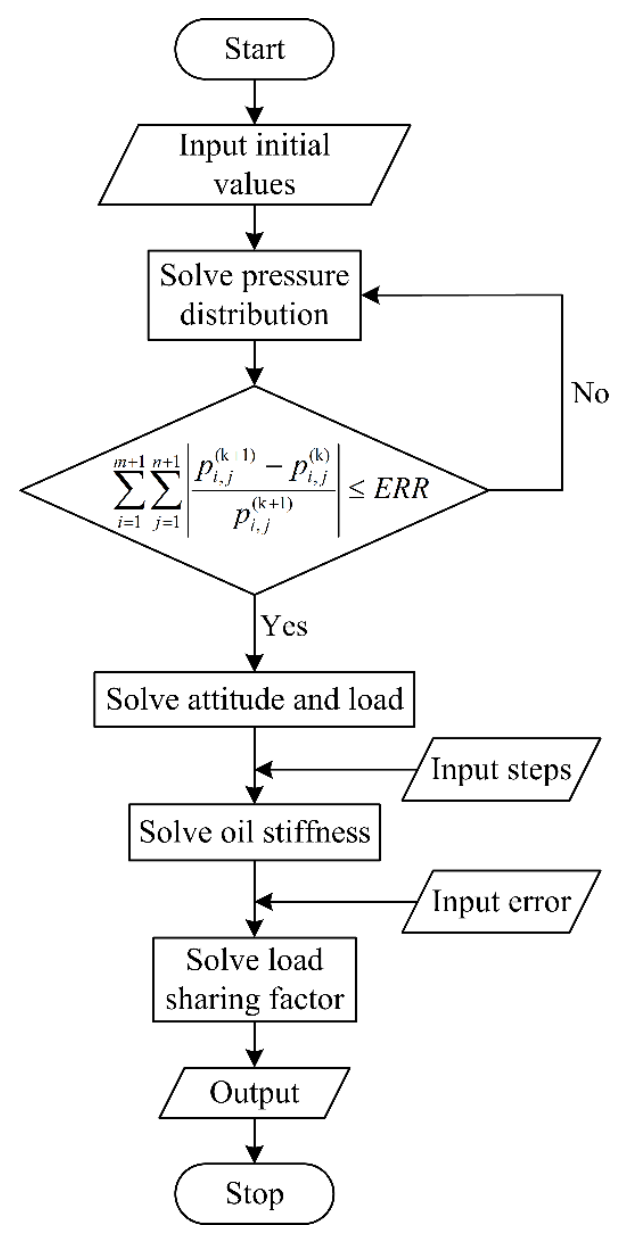

Figure 6. Load sharing factor calculation procedure.

\section{Results and Discussion}

\subsection{Fundamental Characteristics}

An experimental apparatus was built up for the load sharing factor test of a planetary gear set, as shown in Figure 7. In the experimental apparatus, the drive motor provided various torque for the test box of a planetary gear set through a coupling. The input axis was connected to the sun gear. The maximum range of available operating speeds is $0-5000 \mathrm{r} / \mathrm{min}$, which can be precisely controlled. In the experimental planetary gear set, rolling bearings or journal bearings could be applied to the planets alternatively. For the lubrication needs of bearings, a pressure oil supply system was set up, including specified oil distribution sleeves, planet axles with oil paths, the oil inlet, the oil outlet, the oil filter, and an oil pump. The oil pump is capable of supplying an oil flow rate of $40 \mathrm{~L} / \mathrm{min}$, and the maximum oil pressure is about $0.5 \mathrm{MPa}$. A strain gauge was attached to the stationary ring gear for 
measuring the deformation of each planetary gear [30] to quantify the load sharing factor. A data acquisition module was employed to collect and transfer the data to a computer for further analysis.

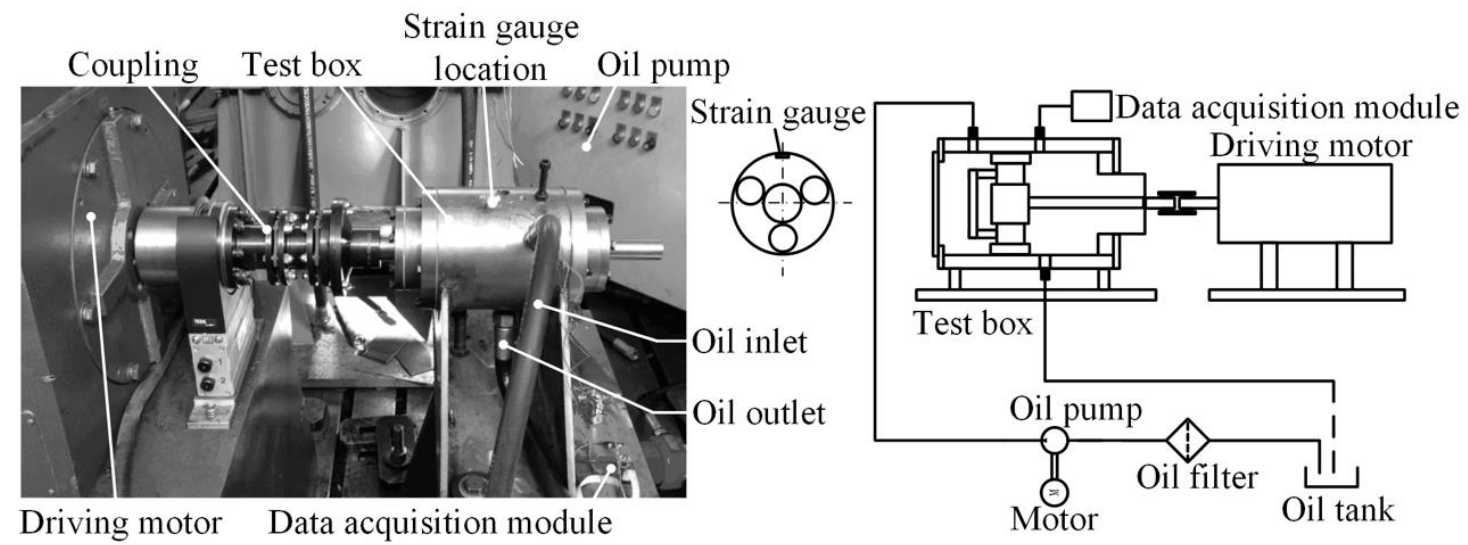

Figure 7. Experimental apparatus.

Figure 8 shows the measured and simulated load sharing factors of a planetary gear set. The maximum sun gear rotation speed used in the experiment was $4000 \mathrm{r} / \mathrm{min}$. As can be seen in the figure, the measured load sharing factor decreased first and then increased along with the increasing rotation speed. The experimental results and the simulated results of the load sharing factors of the planetary gear set with planet journal bearings appeared to have the same trend, along with the changing rotation speeds. The eccentricity ratio of the journal bearings is larger at a lower rotation speed. The large change rate of the load with the eccentricity ratio and the small eccentric angle makes the bearing support stiffness decrease with the increasing speed. However, at a higher rotation speed, the eccentricity ratio becomes smaller. The change rate of the load with the eccentricity ratio also becomes smaller as the eccentric angle becomes larger. As a consequence, the bearing support stiffness increases with the increasing rotation speed. The load sharing factor and the bearing stiffness have the same variation trends with the rotation speed when the load is constant. Obviously, journal bearings affect the variation of the load sharing factor. In addition, the comparison between the applications of rolling bearings and journal bearings in the planetary gear set was also illustrated in the figure. When using planet rolling bearings, the load sharing factors at different rotation speeds were almost the same, which is because the rolling bearing stiffness kept essentially unchanged.

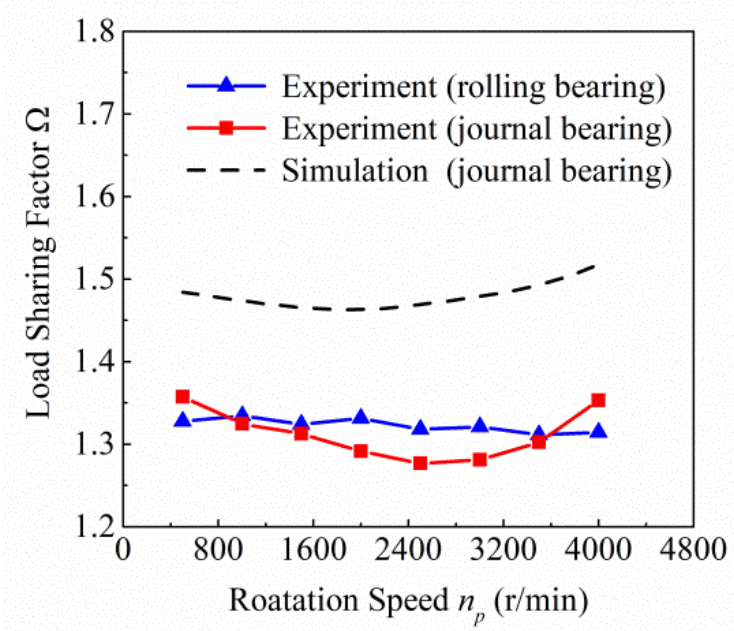

Figure 8. Measured and simulated results of load sharing factors. 


\subsection{Influence Parameters}

The bearing support stiffness of a planetary gear set has a great effect on the load sharing factor [31,32]. The journal bearing stiffness and the load sharing factor are analyzed with different influential parameters. The following figures show the influence of each parameter on the stiffness and the load sharing factor. The structure characteristics of the planetary gear set applied in the computation remain constant. Table 1 gives the structure parameters of the planetary gear set. Table 2 gives the reference parameters in Equations (19)-(27) for estimating the cumulative meshing error.

Table 1. Parameters of the planetary gear set.

\begin{tabular}{cccc}
\hline Parameter & Sun & Planet & Ring \\
\hline Number of planets & - & 4 & - \\
Number of teeth & 48 & 30 & 108 \\
Normal module & 1 & 1 & 1 \\
Pressure angle $\left(^{\circ}\right)$ & 20 & 20 & 20 \\
Helix angle $\left(^{\circ}\right)$ & 20 & 20 & 20 \\
Center distance $(\mathrm{mm})$ & & 39 & \\
\hline
\end{tabular}

Table 2. Reference parameters for meshing errors calculation.

\begin{tabular}{ccc}
\hline Component & Parameter & Value \\
\hline sun & $\beta_{s}$ & $3^{\circ}$ \\
planet & $\beta_{p i}$ & $2^{\circ} / 1^{\circ} / 1.5^{\circ} / 1.5^{\circ}$ \\
carrier & $\beta_{c}$ & $9^{\circ}$ \\
ring & $\beta_{r}$ & $1.8^{\circ}$ \\
sun & $\gamma_{s}$ & $6^{\circ}$ \\
ring & $\gamma_{r}$ & $3.6^{\circ}$ \\
gear & $\alpha_{w}$ & $20^{\circ}$ \\
\hline
\end{tabular}

Figure 9 presents significant variations of the load sharing factor and the journal bearing stiffness with different lengths and diameter ratios of the journal bearings. Both the stiffness and the load sharing factor changed significantly when the lengths and diameter ratio was smaller than 0.75. Under the same rotation speed and load, smaller lengths and diameter ratio increases the eccentricity ratio. When the lengths and diameter ratio becomes larger, the eccentricity ratio also decreases. It seems that the influence of the lengths and diameter ratios is similar to that of the rotation speed. The results also indicate that the load sharing factor is relatively low when the ratio is around 0.7. It is important to choose a suitable ratio to get a proper load sharing factor.

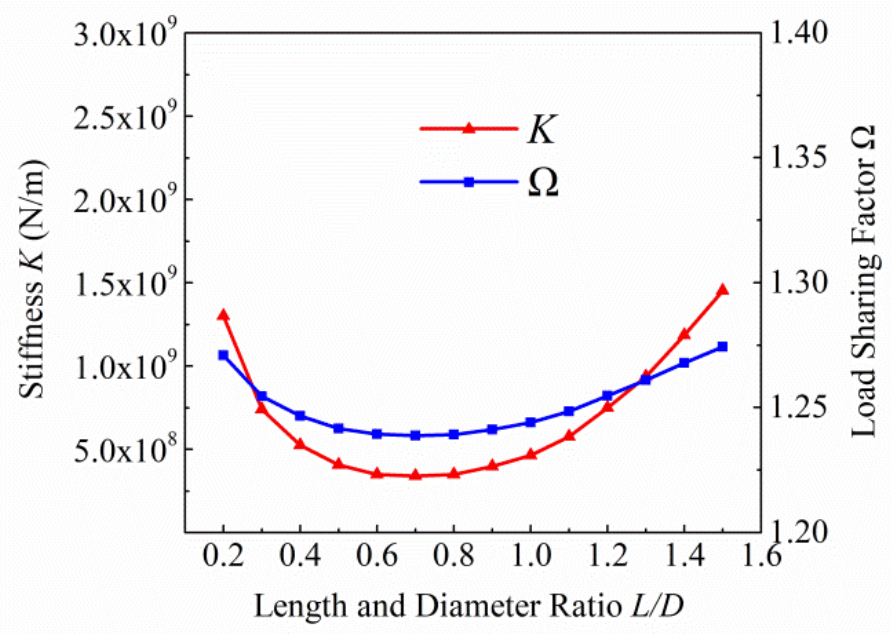

Figure 9. Load sharing factor vs. lengths and diameter ratios. 
Figure 10 illustrates the trends of the load sharing factor and the journal bearing stiffness of the planetary gear with the relative clearance $\psi$, which is calculated by $c / r$. As the relative clearance increased, the stiffness and the load sharing factor both decreased to be stable. When the relative clearance is small, the oil film is thin and the stiffness is large under the same load and rotation speed. As the relative clearance becomes larger, the minimum film thickness will be smaller so as to achieve sufficient load capacity. Thus, the relative clearance should not be too large.

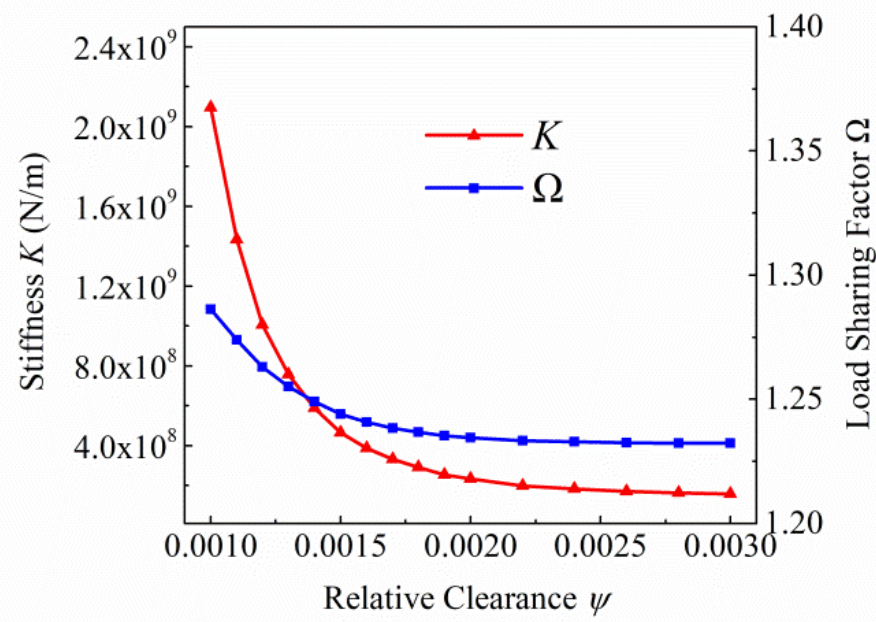

Figure 10. Load sharing factor vs. relative clearance.

Figure 11 shows the load sharing factor and the journal bearing stiffness with different viscosity values. The rotation speed was lower in Figure 11a and was higher in Figure 11b. As the viscosity increased, the bearing stiffness and the load sharing factor increased under the same speed and load. It seems that the load sharing factor reached a minimum value with a small viscosity at the lower rotation speed. As the rotation speed increased, the stiffness and the load sharing factor also became larger. However, the load sharing factor change rate slowed down with a larger viscosity.

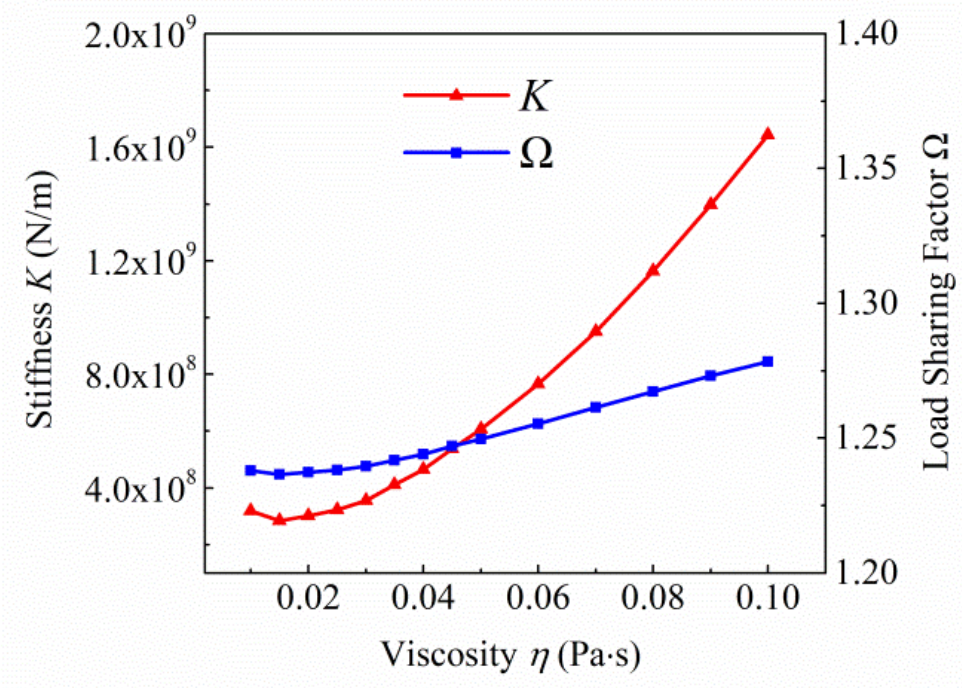

(a)

Figure 11. Cont. 


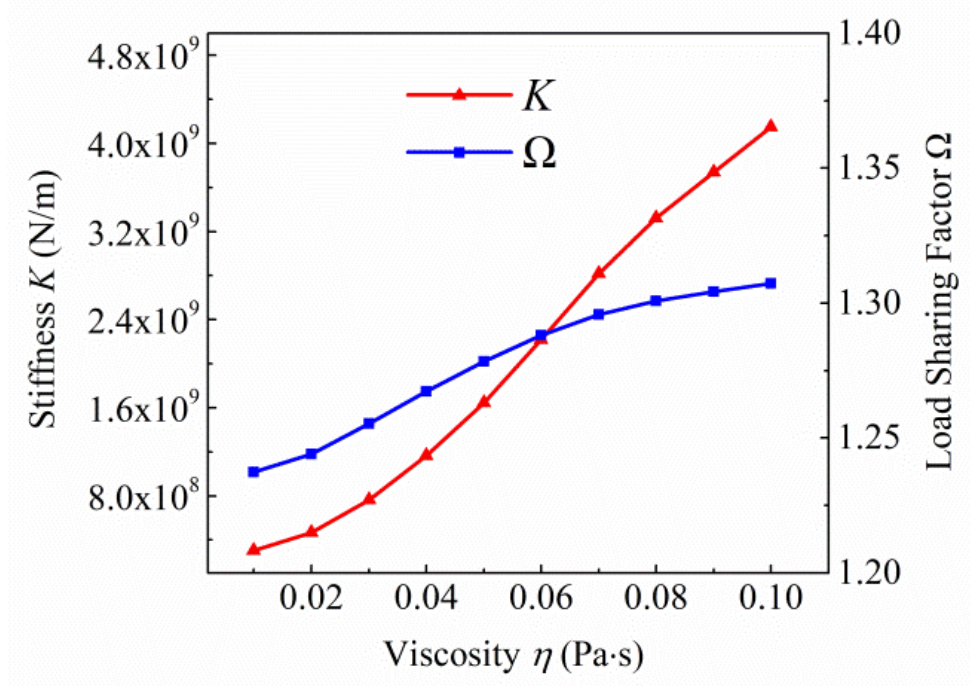

(b)

Figure 11. Load sharing factor vs. viscosity: (a) at a lower rotation speed and (b) at a higher rotation speed.

The variations of the load sharing factor and the bearing stiffness of the load are illustrated in Figure 12. It can be found that the load sharing factor is not only determined by the bearing stiffness. As the load increased, the bearing stiffness increased. However, the load sharing factor decreased to a stable value at last. It is because the planet deformation becomes larger under a higher load. The deformation compensates for the error existing in the gear meshing process and improves the load sharing performance. The influences of the rotation speed on the load sharing factor and bearing stiffness in a high-speed range are presented in Figure 13, which serves as a supplement to Figure 8 to research the effects of rotation speed thoroughly. It seems that the load sharing factor did not increase significantly with the rotation speed in the high-speed range. The growth rate of the load sharing factor slowed down obviously.

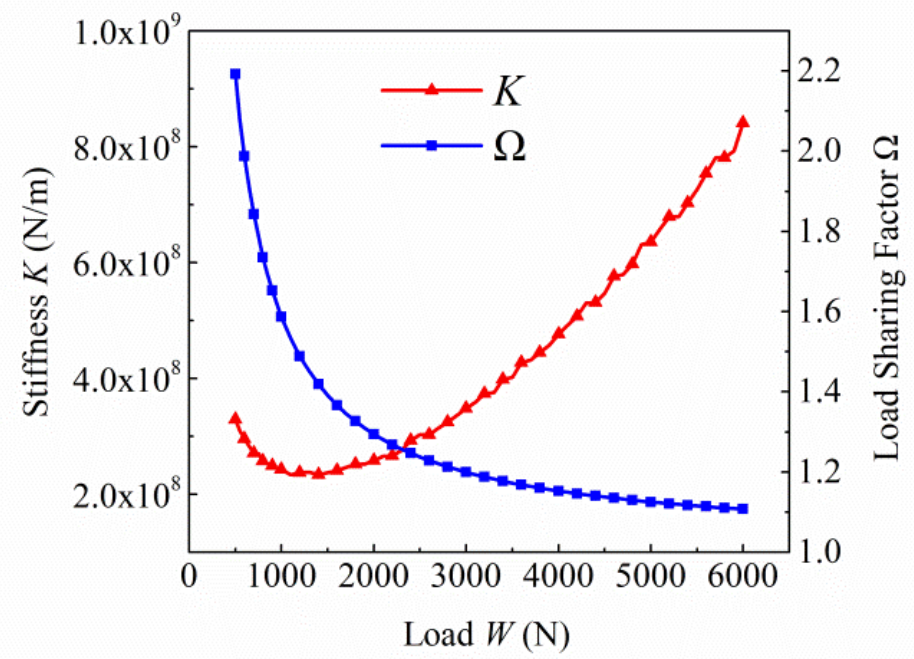

Figure 12. Load sharing factor vs. load. 


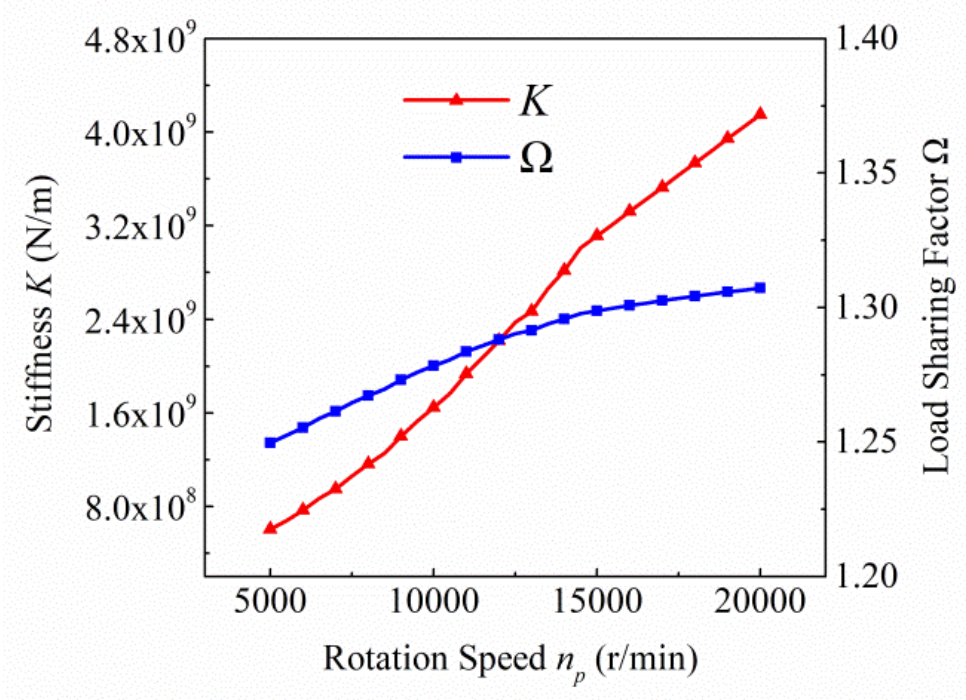

Figure 13. Load sharing factor vs. rotation speed.

\section{Conclusions}

In this study, an available load sharing factor computation method was developed to study the quasi-static load sharing characteristics of a planetary gear set with planet journal bearings. The manufacturing errors, the assembly errors, the floating sun gear error, and the variable journal bearing stiffness were taken into account in the calculation. An experimental apparatus was established. The trend of load sharing factors with changing rotation speeds obtained by simulation was in good agreement with the experimental results, which verified the effectiveness of the proposed load sharing factor computation method. Based on the computation, the effects of journal bearing parameters, including the lengths and diameter ratios and the relative clearance, as well as the effects of operating conditions, including the viscosity, the load, and the rotation speed, were discussed in detail. It can be concluded that the load sharing factor of a planetary gear set with planet journal bearings had a similar variation with the bearing support stiffness under the same load. The load sharing factor became smaller with a larger journal bearing relative clearance or a higher load. There was a minimum load sharing factor with the variation of the length and diameter ratio. As the viscosity increased, the load sharing factor increased under the same rotation speed and load. The load sharing factor change rate slowed down with a large viscosity or at a high rotation speed. These research provided a theoretical basis for the application of journal bearings in planetary gear sets.

For further research, the variations of the journal bearing parameters should be considered as a dynamic process, and the influence on the dynamic behaviors of the planetary gear set should be systematically investigated.

Author Contributions: Conceptualization, W.W.; methodology, Z.G.; validation, Z.G. and L.Z.; data curation, L.Z.; writing —original draft preparation, Z.G.; writing—review and editing, Z.G. and W.W.; and supervision, S.L. and W.W. All authors have read and agreed to the published version of the manuscript.

Funding: This research was funded by the National Natural Science Foundation of China, grant numbers U1864210 and 51975045, and the National Key R\&D Program of China, grant number 2018YFB2001300.

Conflicts of Interest: The authors declare no conflict of interest.

\section{References}

1. Liu, F.; Wu, W.; Hu, J.; Yuan, S. Design of multi-range hydro-mechanical transmission using modular method. Mech. Syst. Signal Process. 2019, 126, 1-20. [CrossRef]

2. Mo, S.; Zhang, Y.; Wu, Q. Load sharing behavior analysis method of wind turbine gearbox in consideration of multiple-errors. Renew. Energy 2016, 97, 481-491. [CrossRef] 
3. Kwon, H.S.; Kahraman, A. An assemblability check methodology for the kinematic configurations of automatic transmission planetary gear trains. J. Mech. Sci. Technol. 2016, 30, 5605-5616. [CrossRef]

4. Yang, F.; Feng, J.; Du, F. Design and power flow analysis for multi-speed automatic transmission with hybrid gear trains. Int. J. Automot. Technol. 2016, 17, 629-637. [CrossRef]

5. Guo, Y.; Keller, J.; LaCava, W. Planetary gear load sharing of wind turbine drivetrains subjected to non-torque loads. Wind Energy 2015, 18, 757-768. [CrossRef]

6. Qiu, X.; Han, Q.; Chu, F. Load-sharing characteristics of planetary gear transmission in horizontal axis wind turbines. Mech. Mach. Theory 2015, 92, 391-406. [CrossRef]

7. Masoumi, A.; Barbieri, M.; Pellicano, F.; Zippo, A.; Strozzi, M. Dynamic imbalance of high-speed planetary gears. Int. J. Cond. Monitor. 2017, 7, 2-6. [CrossRef]

8. Wu, W.; Hu, C.; Hu, J.; Yuan, S. Jet cooling for rolling bearings: Flow visualization and temperature distribution. Appl. Therm. Eng. 2016, 105, 217-224. [CrossRef]

9. Liu, J.; Shao, Y. A new dynamic model for vibration analysis of a ball bearing due to a localized surface defect considering edge topographies. Nonlinear Dyn. 2015, 79, 1329-1351. [CrossRef]

10. Hidaka, T.; Terauchi, Y. Dynamic Behavior of Planetary Gear: 1st Report Load Distribution in Planetary Gear. Bull. JSME 1976, 19, 690-698. [CrossRef]

11. Hayashi, T.; Li, X.Y.; Hayashi, I.; Watanabe, W. Measurement and some discussions on dynamic load sharing in planetary gears. Bull. JSME 1986, 29, 2290-2297. [CrossRef]

12. Kahraman, A. Load sharing characteristics of planetary transmissions. Mech. Mach. Theory 1994, 29, 1151-1165. [CrossRef]

13. Kahraman, A. Static load sharing characteristics of transmission planetary gear sets: Model and experiment. SAE Tech. Paper 1999. [CrossRef]

14. Kahraman, A. Natural modes of planetary gear trains. J. Sound Vib. 1994, 173, 125-130. [CrossRef]

15. Kahraman, A.; Vijayakar, S. Effect of Internal Gear Flexibility on the Quasi-Static Behavior of a Planetary Gear Set. J. Mech. Design 2001, 123, 408-415. [CrossRef]

16. Bodas, A.; Kahraman, A. Influence of carrier and gear manufacturing errors on the static load sharing behavior of planetary gear sets. JSME Int. J. Ser. 2004, 47, 908-915. [CrossRef]

17. Singh, A. Influence of planetary needle bearings on the performance of single and double pinion planetary systems. J. Mech. Design 2007, 129, 85-94. [CrossRef]

18. Fyler, D.C.; Inalpolat, M. A Dynamic Model for Double-Planet Planetary Gearsets. J. Vib. Acoust. 2016, 138, 021006. [CrossRef]

19. Singh, A. Load sharing behavior in epicyclic gears: Physical explanation and generalized formulation. Mech. Mach. Theory 2010, 45, 511-530. [CrossRef]

20. Singh, A. Epicyclic load sharing map-Development and validation. Mech Mach. Theory 2011, 46, 632-646. [CrossRef]

21. Montestruc, A.N. Influence of planet pin stiffness on load sharing in planetary gear drives. J. Mech. Design 2011, 133, 014501. [CrossRef]

22. Gu, X.; Velex, P. A dynamic model to study the influence of planet position errors in planetary gears. J. Sound Vib. 2012, 331, 4554-4574. [CrossRef]

23. Wen, S.Z. Tribology Theory; Publishing Company of Tisinghua University: Beijing, China, 1991.

24. Yu, R.; Chen, W.; Li, P. The analysis of elastohydrodynamic lubrication in the textured journal bearing. Proc. Int. Mech. Eng. J. 2016, 230, 1197-1208. [CrossRef]

25. Ren, F.; Luo, G.; Shi, G.; Wu, X.; Wang, N. Influence of manufacturing errors on dynamic floating characteristics for herringbone planetary gears. Nonlinear Dyn. 2018, 93, 361-372. [CrossRef]

26. Zhai, H.; Zhu, C.; Song, C.; Liu, H.; Bai, H. Influences of carrier assembly errors on the dynamic characteristics for wind turbine gearbox. Mech. Mach. Theory 2016, 103, 138-147. [CrossRef]

27. Hu, Y.; Talbot, D.; Kahraman, A. A Load Distribution Model for Planetary Gear Sets. J. Mech. Design 2018, 140, 053302. [CrossRef]

28. Ligata, H.; Kahraman, A.; Singh, A. A closed-form planet load sharing formulation for planetary gear sets using a translational analogy. J. Mech. Design 2009, 131, 021007. [CrossRef]

29. Xiao, T.; Yuan, S. Analysis on the performance of load sharing mechanism by oil film floated in planetary gear. Chin. J. Mech. Eng. 1993, 29, 75-81. 
30. Ligata, H.; Kahraman, A.; Singh, A. An Experimental Study of the Influence of Manufacturing Errors on the Planetary Gear Stresses and Planet Load Sharing. ASME J. Mech. Des. 2008, 130, 041701. [CrossRef]

31. Iglesias, M.; del Rincon, A.F.; de-Juan, A.; Garcia, P.; Diez-Ibarbia, A.; Viadero, F. Planetary transmission load sharing: Manufacturing errors and system configuration study. Mech. Mach. Theory 2017, 111, 21-38. [CrossRef]

32. Zhang, H.; Wu, S.; Peng, Z. A nonlinear dynamic model for analysis of the combined influences of nonlinear internal excitations on the load sharing behavior of a compound planetary gear set. Proc. Int. Mech. Eng. Part C 2016, 230, 1048-1068. [CrossRef]

C 2020 by the authors. Licensee MDPI, Basel, Switzerland. This article is an open access article distributed under the terms and conditions of the Creative Commons Attribution (CC BY) license (http://creativecommons.org/licenses/by/4.0/). 